\title{
Development of a novel
} immunochromatographic lateral flow assay specific for Mycobacterium bovis cells and its application in combination with immunomagnetic separation to test badger
faeces

Linda D. Stewart ${ }^{1}$, Nuria Tort ${ }^{2}$, Paul Meakin², Jose M. Argudo ${ }^{1}$, Ruramayi Nzuma ${ }^{1}$, Neil Reid ${ }^{1,3}$, Richard J. Delahay ${ }^{4}$, Roland Ashford ${ }^{5}$, W. lan Montgomery ${ }^{1,3}$ and Irene R. Grant ${ }^{1 *}$ (i)

\begin{abstract}
Background: The European badger is an important wildlife reservoir of Mycobacterium bovis implicated in the spread of bovine tuberculosis in the United Kingdom and Ireland. Infected badgers are known to shed $M$. bovis in their urine and faeces, which may contaminate the environment. To aid bovine tuberculosis control efforts novel diagnostic tests for detecting infected and shedding badgers are needed. We proposed development of a novel, rapid immunochromatographic lateral flow device (LFD) as a non-invasive test to detect M. bovis cells in badger faeces. Its application in combination with immunomagnetic separation (IMS) to detect Mycobacterium bovis cells in badger faeces is reported here.

Results: A novel prototype LFD for M. bovis cells was successfully developed, with unique specificity for M. bovis and a limit of detection $50 \%\left(\mathrm{LOD}_{50 \%}\right)$ of $1.7 \times 10^{4} \mathrm{M}$. bovis cells $/ \mathrm{ml}$. When IMS was employed to selectively capture and concentrate $M$. bovis cells from badger faeces prior to LFD testing, the LOD $50 \%$ of the IMS-LFD assay was $2.8 \times 10^{5} \mathrm{M}$. bovis cells $/ \mathrm{ml}$ faecal homogenate. Faeces samples collected from latrines at badger setts in a region of endemic bovine tuberculosis infection were tested; 78 (18\%) of 441 samples tested IMS-LFD assay positive, whereas 140 (32\%) tested IMS-qPCR positive (Kappa agreement $-0.009 \pm 0.044, p=0.838$ ). Subsequently, when 130 faeces samples from live captured, or captive, badgers of known infection status (on the basis of StatPak, interferon- $\gamma$ and/or culture results) were tested, the IMS-LFD assay had higher relative diagnostic specificity (Sp 0.926), but poorer relative diagnostic sensitivity (Se 0.081), than IMS-qPCR (Sp 0.706, Se 0.581) and IMS-culture (Sp 0.794, Se 0.436).

Conclusions: The novel IMS-LFD assay, although very specific for $M$. bovis, has low analytical sensitivity (indicated by the $\left.L_{0 D}{ }_{50 \%}\right)$ and would only detect badgers shedding high numbers of $M$. bovis $\left(>10^{4-5} \mathrm{cell} / \mathrm{s} / \mathrm{g}\right)$ in their faeces. The novel LFD would, therefore, have limited value as a non-invasive test for badger TB surveillance purposes but it may have value for alternative veterinary diagnostic applications.
\end{abstract}

Keywords: Mycobacterium bovis, European badger (Meles meles), Faeces, immunomagnetic separation, Lateral flow device, Non-invasive test, Diagnostic specificity, Diagnostic sensitivity

\footnotetext{
*Correspondence: i.grant@qub.ac.uk

${ }^{1}$ Institute for Global Food Security, School of Biological Sciences, Queen's

University Belfast, Belfast, Northern Ireland, UK

Full list of author information is available at the end of the article
} 


\section{Background}

Mycobacterium bovis is the causative agent of bovine tuberculosis (TB) in cattle, badgers and other wild and domestic mammals [1]. Bovine TB is the most serious endemic disease currently facing the livestock industry in the United Kingdom and Republic of Ireland [2]. Despite a systematic test and slaughter programme in cattle, which has been ongoing for many years within the United Kingdom, the incidence of bovine TB has continued to rise. This lack of success has been attributed in part to the presence of a reservoir of infection in the European badger (Meles meles) [3, 4]. Investigation of the dynamics of TB in wildlife and cattle herds using DNA fingerprinting of strains $[5,6]$ has revealed that badgers and cattle tend to have similar $M$. bovis spoligotypes, providing evidence of cross-species transmission [7]. In parts of the United Kingdom, herd incidence rates of TB in cattle more than doubled between 1998 and 2010, and although prevalence varies widely in badger populations, estimates of 6.3-33.9\% infection (depending on county, overall 16.6\%) were recorded in 2005 in endemic areas [3]. The ability to identify and monitor TB infection in the reservoir wildlife host may provide a valuable tool for targeting efforts to control risks of $M$. bovis transmission to cattle.

It is known that badgers can transmit $M$. bovis to cattle [8], but the precise route of transmission is unclear. A potential route if badgers and cattle come into close proximity is inhalation of aerosolised droplets; however, infected badgers are also known to shed M. bovis in their urine and faeces $[9,10]$, which may contaminate the environment. Badgers habitually defecate in clusters of shallow pits known as latrines which if accessible to cattle may act as a possible source of infection [11]. Infected badgers are known to intermittently shed $M$. bovis in their faeces in variable numbers. For example, King et al. [12] demonstrated heterogeneity in bacterial load for badgers in the Woodchester Park, Gloucestershire, study area, and reported shedding of $1 \times 10^{3}-4 \times 10^{5} \mathrm{M}$. bovis cells per $\mathrm{g}$ of faeces. Faecal shedding is an indication of infectiousness, with shedding correlating with animals exhibiting more severe disease status [13]. M. bovis can persist in the environment for several months under certain conditions [14]. This environmental signal of the presence of $M$. bovis in a badger population was demonstrated by Courtenay et al. [15] who showed that the detectability of the bacterium at badger setts and latrines was strongly linked to the frequency of excretion detected in live-sampled badgers.

The M. bovis infection status of badgers in a given area may be assessed in various ways: by active surveillance involving the capture, anaesthesia and use of blood tests and microbiological culture of samples (invasive); by collecting and analysing badger faeces or other environmental samples (non-invasive); or, by the examination of badger carcasses after road traffic accidents (RTA) and tissue culture. The appropriate method to adopt depends on the question being asked and the geographical scale for which data are required. For example, RTA surveys have been used to provide coarse prevalence (or even presence/ absence) data on a large geographical scale [16-19], whereas live capture and collection of clinical samples has been used to provide more detailed eco-epidemiological data over smaller areas [20]. The collection of faecal samples from latrines for TB surveillance in badgers has the potential to be used on a variety of geographical scales.

Since it is known that some infected badgers excrete $M$. bovis in their faeces $[9,10]$, we proposed the development of an immunochromatographic lateral flow device (LFD) and its use in combination with an existing immunomagnetic separation (IMS) technique $[21,22]$ as a non-invasive test for the presence of $M$. bovis. Immunochromatographic assays are easy to use, cheap to produce, and provide a rapid result (within $15 \mathrm{~min}$ ), so are ideal for field use. The purpose of including IMS was to selectively capture and concentrate target mycobacterial cells from the faeces matrix before application to the LFD, to facilitate sample clean-up and improve detection sensitivity. Here, we report the successful development of a prototype $M$. bovis-specific LFD and its application in combination with IMS to test for evidence of $M$. bovis cells in badger faeces. We also assessed agreement between results of the field IMS-LFD assay and laboratory IMS-based methods (IMS-qPCR and IMS-culture) when faeces from badgers of unknown infection status were tested, and estimated relative diagnostic sensitivity (Se) and specificity (Sp) of the IMS-LFD assay by testing faeces from badgers for which we had independent information on their $M$. bovis infection status.

\section{Methods}

Bacterial cultures employed in the study

Mycobacterium bovis AF2122/97, M. bovis BCG NCTC 5692, M. avium subsp. avium NCTC 13034, M. avium subsp. paratuberculosis ATCC 19698, M. fortuitum NCTC 10394, M. intracellulare NCTC 10425, M. kansasii NCTC 10268, M. xenopi NCTC 10042, M. terrae NCTC 10856, M. scrofulaceum NCTC 10803, M. marinum NCTC 2275, M. smegmatis mc ${ }^{2}$ 155, M. gordonae NCTC 10267, M. tuberculosis H37Rv, and a field isolate of M. hiberniae were cultured in Middlebrook 7H9 broth containing 10\% OADC supplement (both Difco) to stationary phase, harvested by centrifugation and washed in phosphate buffered saline, pH 7.2 (PBS, Sigma). Cell suspensions in PBS, containing $10^{6}-10^{7} \mathrm{CFU} / \mathrm{ml}$, were subjected to a $10 \mathrm{kGy}$ dose of gamma radiation using a Gammabeam 650 cobalt irradiator, in order to kill the bacteria without damaging cell surface antigens and to render them safe to use in a Containment Level 2 laboratory. Irradiated PBS bacterial suspensions were stored at $-80{ }^{\circ} \mathrm{C}$ until required. 
In addition, colonies of $M$. tuberculosis H37Rv grown on solid agar were emulsified in PBS and $80 \mu$ tested directly on the LFD in a Containment Level 3 laboratory.

\section{Development of a prototype lateral flow device (LFD) specific for M. bovis}

A selection of previously produced [21] or locally sourced M. bovis-specific monoclonal and polyclonal antibodies was tested for their suitability for incorporation into the proposed LFD. In order to select the most appropriate antibodies to serve as capture and detector reagents, a series of trials were carried out. All the antibodies were conjugated to gold nanoparticles and also all were immobilised at the Test $(\mathrm{T})$ line of different nitrocellulose membranes at different concentrations. A cocktail of commercial antimouse-IgG IgG and anti-rabbit-IgG IgG was immobilized at the Control (C) Line position. A two-step wet assay was performed to evaluate recognition of $M$. bovis whole cells using the different combinations of membranes (with the immobilized antibodies in the T Line) and gold conjugates. The combination of antibodies and membrane which gave the strongest T-line was selected in order to produce a batch of working prototype devices to be evaluated.

\section{Assessment of detection specificity and cross-reactivity of the prototype LFD}

PBS suspensions of the range of Mycobacterium spp. identified above and six different spoligotypes of $M$. bovis (SB140 (AF2122/97), SB129, SB273, SB142, SB263 and SB145) prepared as described above, were tested on the prototype LFD. Irradiated cultures were vortexed briefly before $80 \mu \mathrm{l}$ were transferred to the sample well of the LFD. The presence or absence of a T-line on the LFD after $15 \mathrm{~min}$ at room temperature was assessed visually in each case.

\section{Determination of the limit of detection of the prototype LFD}

The $50 \%$ limit of detection $\left(\mathrm{LOD}_{50 \%}\right)$ of the LFD was determined by testing 10-fold serial dilutions of a stock of irradiated M. bovis AF2122/97 (containing $5.3 \times 10^{6} \mathrm{CFU} / \mathrm{ml}$ ) in PBS. Four replicate samples at each of four dilutions containing $10^{5}, 10^{4}, 10^{3}$ and $0 \mathrm{CFU} / \mathrm{ml}$ were tested. The presence or absence of a T-line was assessed both visually and using an LFD reader (Forsite Diagnostics Limited, York, UK) after the sample had been run on the LFD for $15 \mathrm{~min}$ at room temperature. The $\mathrm{LOD}_{50 \%}$ was determined using the generalized Spearman-Kärber LOD $_{50 \%}$ calculation for 4-level spiking protocols [23].
Coating of magnetic beads for immunomagnetic separation (IMS) and determination of $M$. bovis capture sensitivity and bead specificity

The MyOne tosylactivated Dynabeads (Life Technologies, Paisley, UK) previously used by Stewart et al. [22] for immunocapture of $M$. bovis from bovine lymph node tissue are $1 \mu \mathrm{m}$ in diameter. These proved to be too large to run along the LFD, so smaller carboxylated magnetic beads, available in three sizes (200, 300 and $500 \mathrm{~nm}$ ) were sourced (Ademtech, France). All three sizes of Ademtech bead were coated with the M. bovis-specific 11G3 monoclonal antibody and EEA302 biotinylated peptide [21] separately, and also with a mixture of the peptide and the antibody (dually coated), using the Carboxyl-Adembeads Coupling Kit (Ademtech 02820) according to the manufacturer's instructions. Tenfold serial dilutions $\left(10^{-1}\right.$ to $\left.10^{-5}\right)$ of irradiated $M$. bovis AF2122/97 $\left(10^{6}-10^{7} \mathrm{CFU} / \mathrm{ml}\right)$ were prepared and $1 \mathrm{ml}$ of each dilution was subjected to automated IMS using the Dynal BeadRetriever (Life Technologies) (21) using the different sized/coated Adembeads, with resuspension of the beads after IMS in $100 \mu \mathrm{l}$ Tris-EDTA (TE) buffer. After extraction of DNA by heating at $100{ }^{\circ} \mathrm{C}$ for $25 \mathrm{~min}$, the samples were analysed using $M$. bovis touchdown PCR [24] and visualised using agarose gel electrophoresis PCR, as described in Stewart et al. [21]. As controls, and to enable comparison of capture sensitivities achieved, dually coated MyOne Tosylactivated Dynabeads (Life Technologies) and a dilution series of irradiated M. bovis AF2122/97 in TE buffer not subjected to IMS were included.

Specificity of the 200 and $300 \mathrm{~nm}$ coated beads was assessed by IMS experiments involving the range of Mycobacterium spp. described above. Each species was tested on two separate occasions. Stationary phase broth cultures of each species were diluted to $10^{3}-10^{4} \mathrm{CFU} / \mathrm{ml}$ in Middlebrook 7H9/OADC broth. A $100 \mu \mathrm{l}$ sample of the dilutions was spread onto Middlebrook 7H10/OADC agar plates to determine CFU/ml before IMS and $1 \mathrm{ml}$ of each dilution was then subjected to automated IMS using each of the coated beads. Following IMS, beads were resuspended in $1 \mathrm{ml}$ Middlebrook 7H9/OADC broth (maintaining original sample volume in order to permit direct comparison of colony counts before and after IMS), $100 \mu \mathrm{l}$ of which was spread onto Middlebrook 7H10/OADC agar plates. Agar plates were incubated at 30,37 or $42{ }^{\circ} \mathrm{C}$ (as appropriate for the Mycobacterium sp. concerned) until colonies were evident. Mean colony count following IMS was expressed as a percentage of the number of CFU present in the original suspension before IMS to calculate the degree of non-specific binding by Mycobacterium spp. other than M. bovis.

\section{Optimisation of faeces sample preparation protocol}

Optimisation of the faeces sample preparation protocol is described in detail elsewhere [25]. Briefly, a number of 
factors in relation to application of IMS in the field needed to be considered before arriving at a finalised field IMS protocol. These included the volume of diluent required to homogenise the faecal samples, the effect of the faecal matrix on bead retrieval, the time needed to retrieve the beads, the dilution factor required, the quantity of beads required, the assessment of matrix effect on capture of $M$. bovis by coated beads, and the effect of nylon strainers on the recovery of $M$. bovis.

\section{Limit of detection of IMS-LFD assay applied to faeces}

The $\mathrm{LOD}_{50 \%}$ of the IMS-LFD assay was determined twice using two different $M$. bovis negative badger faeces samples as the spiking matrix. Four replicate samples were spiked at four cell concentrations $\left(10^{5}, 10^{4}, 10^{3}\right.$ and $0 \mathrm{CFU} / \mathrm{ml}$ ) before the field IMS-LFD assay was applied. The presence or absence of T-lines was assessed visually. The $\mathrm{LOD}_{50 \%}$ was, once again, estimated using the generalized Spearman-Kärber LOD $_{50 \%}$ calculation for 4-level spiking protocols [23].

\section{Ability of the novel IMS-LFD assay to detect $M$. bovis in naturally infected badger faeces}

Badger faeces samples were collected from latrines at 110 setts throughout Northern Ireland. A random sample of $1 \mathrm{~km}$ squares containing main setts, located during the Northern Ireland Badger Survey 2007/08 [26], were selected to be visited for sampling purposes. These setts were located in geographic areas with high, moderate and low reported incidence of tuberculosis breakdowns in cattle, based on information supplied by the Department of Agriculture and Rural Development for Northern Ireland. On arrival at each main sett, a single latrine was located from which up to a maximum of five of the freshest appearing faecal samples were collected; each sample was taken from a different dropping.

The IMS-LFD procedure was performed in the field as summarised schematically in Fig. 1. Approximately $1 \mathrm{~g}$ of badger faeces was transferred to a tube containing $9 \mathrm{ml} \mathrm{PBS} \mathrm{pH} 7.2$ and the sample was shaken vigorously by hand. When a homogeneous suspension was obtained, the sample was filtered through a $70 \mu \mathrm{m}$ cell strainer (Falcon) into a $50 \mathrm{ml}$ centrifuge tube. A sub-sample of $6-8 \mathrm{ml}$ of this homogenised, filtered faeces sample was poured into a tube containing $20 \mu \mathrm{l}$ of antibody- and peptide- dually coated $300 \mathrm{~nm}$ carboxylated magnetic beads. The sample was incubated at ambient temperature for $30 \mathrm{~min}$ and shaken every $5 \mathrm{~min}$. The tube was placed in a DynaMag ${ }^{\mathrm{mm}}-$ 15 magnetic rack (Life Technologies) for 10 min before the supernatant was carefully poured off, leaving the beads behind. Beads were washed three times by shaking in approx. $5 \mathrm{ml}$ PBS-0.05\% Tween 20 (PBS-T, Sigma) per wash with separation for 2 min on the magnetic rack between washes. After the third wash the beads were resuspended in $200 \mu \mathrm{l}$ Detector ${ }^{\mathrm{rm}}$ block solution (KPL Inc., Gaithersburg, USA) and $80 \mu \mathrm{l}$ was transferred to the test well of the LFD. After $15 \mathrm{~min}$ the IMS-LFD result was recorded as 'positive' if both Control (C) and Test (T) lines were visible or 'negative' if only the $\mathrm{C}$ line was observed. Digital photographs were taken to record test outcomes. The LFDs were retained and returned to the laboratory where the presence of a $\mathrm{T}$ line was subsequently verified using a Forsite LFD reader. Residual bead suspensions were also returned to the laboratory for Ziehl-Neelsen staining and examination by light microscopy, in order to verify the presence of large numbers of acid-fast cells in samples which had tested IMS-LFD positive in the field.

\section{Laboratory IMS-based tests on residual faecal homogenates}

The residual 1-2 ml portion of each homogenised, filtered badger faecal sample was returned to the laboratory where automated IMS followed by real-time qPCR (IMS-qPCR) and MGIT $^{\mathrm{Tm}}$ culture (IMS-culture) were carried out. One $\mathrm{ml}$ of each homogenised, filtered badger faecal sample was subjected to automated IMS with dually antibody- and peptide-coated MyOne tosylactivated Dynabeads [21] using a Dynal BeadRetriever (both Life Technologies). Following automated IMS, the beads plus any captured $M$. bovis cells were resuspended in $500 \mu$ l Middlebrook 7H9 broth which was then split between a $100 \mu \mathrm{l}$ sample for qPCR (method of Sweeney et al. [27]) and $400 \mu \mathrm{l}$ for BACTEC ${ }^{\mathrm{Tm}}$ MGIT $^{\mathrm{m}}$ culture (Becton Dickinson). DNA was released from beadbound $M$. bovis cells by boiling for $25 \mathrm{~min}$ and then purified by Zymoclean columns (ZymoResearch) before qPCR was performed using an Eco-PCR instrument (Illumina, Inc.). The reaction volume was $25 \mu \mathrm{l}$ comprising $12.5 \mu \mathrm{l}$ of TaqMan Gene expression $\times 2$ master mix (Life Technologies), $1 \mu \mathrm{l}(20 \mathrm{pmol} / \mu \mathrm{l})$ forward RD4 flanking primer (5' TGTGAATTCATACAAGCCGTAGTCG 3'), $1 \mu \mathrm{l}(20 \mathrm{pmol} / \mu \mathrm{l})$ reverse RD4 flanking primer (5' CCCGTAGCGTTACTGAGAAATTGC $\left.3^{\prime}\right), \quad 1 \quad \mu \mathrm{l}$ (10 pmol/ $\mu \mathrm{l})$ RD4 hydrolysis probe (5' 6-FAM-AGCG CAACACTCTTGGAGTGGCCTAC-MGB 3'), $7 \mu \mathrm{l}$ of nuclease-free sterile water and $2.5 \mu \mathrm{l}$ of template DNA. The RD4 hydrolysis probe and qPCR primers were purchased from Life Technologies. Reaction conditions were: $50{ }^{\circ} \mathrm{C}$ for $2 \mathrm{~min}, 95{ }^{\circ} \mathrm{C}$ for $10 \mathrm{~min}$, and 40 cycles of $95{ }^{\circ} \mathrm{C}$ for $15 \mathrm{~s}$ and $60^{\circ} \mathrm{C}$ for $1 \mathrm{~min}$. Duplicate $2.5 \mu \mathrm{l}$ aliquots of DNA were tested for each faecal sample. Both aliquots had to report positive or negative for a definitive IMS-qPCR result to be recorded. In instances where there was disagreement between the duplicate results for any sample, the qPCR was repeated. If disagreement between duplicate results still existed after the second PCR then the sample was recorded as IMS-qPCR negative (interpretation adopted by Travis et al. [28]). A six point standard curve, generated 


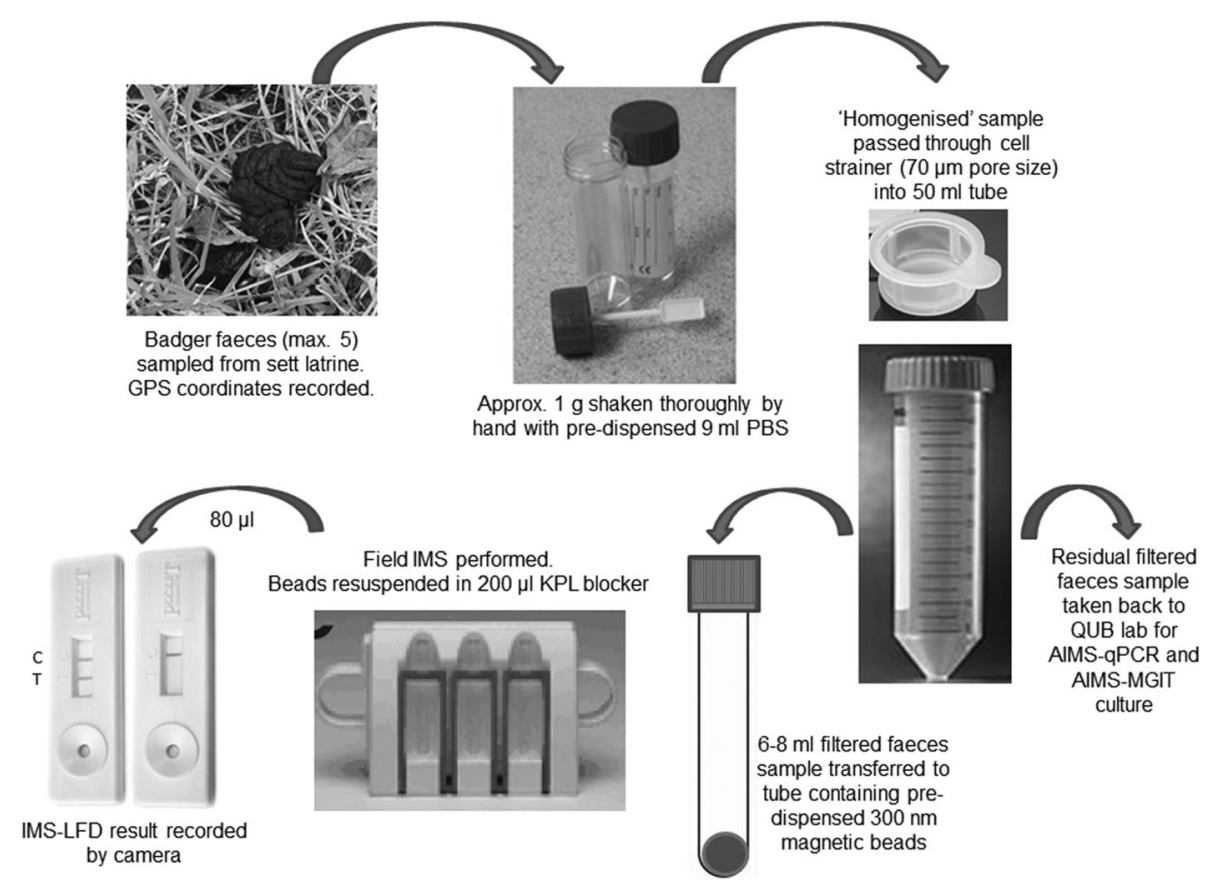

Fig. 1 Schematic outlining the field IMS-LFD testing procedure for testing badger faeces

using DNA from a dilution series $\left(10^{6}-10 \mathrm{CFU} / \mathrm{ml}\right)$ of irradiated M. bovis AF2122/97, and a no template control (water only), were run in duplicate in each qPCR run.

For IMS-culture, after IMS the beads $(400 \mu \mathrm{l})$ were inoculated directly into BACTEC ${ }^{\mathrm{m} x}$ MGIT $^{\mathrm{Tn}}$ culture tubes supplemented with 10\% OADC supplement and PANTA antibiotic supplement (all Becton Dickinson). The MGIT cultures were observed visually (because no MGIT 960 instrument was available in the laboratory) at intervals over an incubation period of up to 12 weeks at $37^{\circ} \mathrm{C}$ for signs of growth (turbidity change). After 4, 8 and 12 weeks of incubation $100 \mu \mathrm{l}$ of each MGIT culture showing evidence of growth was removed, boiled at $100{ }^{\circ} \mathrm{C}$ for $25 \mathrm{~min}$ and checked for presence of $M$. bovis DNA by Touchdown PCR targeting the IS6110 element and employing INS1 and INS2 primers [24]. The Touchdown PCR was able to detect down to $50 \mathrm{M}$. bovis CFU/ml (Stewart and Grant, unpublished data). An IMS-culture positive result was declared if MGIT cultures showing evidence of growth (turbidity) tested Touchdown PCR positive. If no growth was observed after 12 weeks or if the final Touchdown PCR results for the MGIT positive cultures at 12 weeks were all negative, then a negative IMS-culture result was declared.

Assessment of the diagnostic specificity and sensitivity of the IMS-LFD assay by testing faeces from badgers of putative known infection status

In total, 130 faecal samples were collected from badgers for which independent live animal TB diagnostic test results were also available. The samples were tested by the IMS-LFD assay, IMS-qPCR and IMS-culture, as described above for the badger faecal samples collected from latrines, except that sample preparation and the IMS-LFD test were performed in the laboratory. Of the 130 faecal samples, 100 had been obtained, following administration of an enema, from badgers trapped as part of the long-term capture-mark-release project at Woodchester Park, Gloucestershire, England. In order to maximise the likelihood that a proportion of these samples came from $M$. bovis-infected animals, we targeted collection at badger social groups with evidence of current or recent live animal test positive results. Following collection all faeces samples were stored at $-70{ }^{\circ} \mathrm{C}$ for up to 7 months prior to testing. BrockTB Stat-Pak (Chembio Diagnostic Systems, Inc., Medford, NY), culture (of sputum, faeces, urine, wound/abscess swabs), and interferon-gamma enzyme immunoassay (IFN- $\gamma$ EIA [29]) test results, obtained over the previous $1-2$ years, were available for these animals. The remaining 30 faecal samples were obtained from captive badgers that were originally trapped in a part of the United Kingdom with very low incidence of TB in cattle. These 30 badgers had tested negative for $M$. bovis infection by the IFN- $\gamma$ EIA test and bacteriological culture of clinical samples (sputum, urine and rectal swab) on three sequential occasions over a three month period. They were, therefore, considered putative 'non-infected'. All 130 faeces samples were blind tested by staff at Queen's University Belfast. 


\section{Statistical analysis}

Descriptive statistics captured the prevalence of $M$. bovis positive results using the three IMS-based tests (IMSLFD, IMS-qPCR and IMS-culture) on 441 badger faeces of unknown disease infection status collected in Northern Ireland, and 130 badger faecal samples collected from Woodchester Park $(n=100)$ and captive $(n=30)$ animals for which contemporaneous or previous live animal diagnostic test results were available. Venn diagrams were prepared to illustrate inter-relationships between the results obtained with each test. Cross-tabulation of results permitted determination of Kappa statistics, as a measure of the agreement between test results, which were interpreted according to Landis and Koch [30]. McNemar's test was performed on $2 \times 2$ contingency tables of results for the three IMS-based tests versus the badgers' putative TB infection status derived on the basis of the live animal diagnostic test results, to permit estimation of diagnostic sensitivity (Se) and specificity (Sp) of the field IMS-LFD, IMS-qPCR and IMS-culture tests applied to faeces. Since no gold standard diagnostic test for $M$. bovis in badgers exists, estimates of diagnostic specificity and sensitivity are relative to the live animal tests applied. All statistical analyses were performed using SPSS 21 (IBM), unless otherwise stated.

\section{Results}

Development of a prototype $M$. bovis-specific LFD

A specific monoclonal mouse anti-M. bovis antibody (Mab) and a rabbit anti-M. bovis polyclonal antibody (Pab) were successfully conjugated to the gold nanoparticles, and the resulting conjugates were found to be stable. Potential capture reagents were immobilised at the T-line position on the membranes and a cocktail of commercial anti-mouse-IgG and anti-rabbit-IgG IgG was immobilized at the C-line position. Following trials with different combinations and concentrations of capture and detector reagents, it was concluded that the working prototype LFD for the detection of $M$. bovis whole cells was comprised of 'membrane 1' with polyclonal antibody immobilised at the T-Line to act as the capture reagent and Mab conjugated to gold nanoparticles to act as the detector reagent. A batch of 1000 prototype LFDs was produced by Forsite Diagnostics Ltd., York, for evaluation at Queen's University Belfast. For commercial reasons, no additional detail about the membrane or antibodies used for the prototype LFD device can be provided here.

When the detection specificity of the prototype LFD was evaluated by testing a range of different Mycobacterium spp. diluted in PBS, the prototype LFD yielded a positive $\mathrm{T}$-line for $M$. bovis (all six spoligotypes tested) and $M$. bovis BCG only (Fig. 2a), and gave a negative T-line with all other Mycobacterium spp. tested (Fig. 2b). In terms of detection sensitivity, the
$\mathrm{LOD}_{50 \%}$ for $M$. bovis suspended in PBS was determined to be $1.7 \times 10^{4} \mathrm{M}$. bovis cells $/ \mathrm{ml}$.

\section{IMS modification}

Evaluation of the smaller Ademtech carboxylated magnetic beads indicated that, like the previously used MyOne Tosylactivated beads, dually coated beads (i.e. coated with both peptide and IgM antibody) produced the best $M$. bovis capture results. The dually coated $300 \mathrm{~nm}$ and $200 \mathrm{~nm}$ beads showed similar capture capability from both buffer and spiked faeces, with $<3 \%$ non-specific binding with all the other non-target mycobacteria tested. It was, therefore, concluded that either sized bead could be used with the LFD, and we decided to use the $300 \mathrm{~nm}$ beads.

\section{Combining field IMS with the LFD}

After field IMS the beads were resuspended in $200 \mu \mathrm{l}$ PBS for analysis on the LFD. However, it was found that uncoated beads, coated beads and the storage buffer recommended by Ademtech all produced a false positive T-line on the LFD after IMS. To resolve this issue an extra step of quenching the activated unused carboxyl groups present on the bead surface using ethanolamine was introduced during coating of the beads, and various blocking solutions were investigated as alternatives to the Ademtech storage buffer for resuspension of the coated beads. The false positive issue was resolved by resuspension of beads after IMS in KPL Detector ${ }^{\mathrm{TM}}$ block, a commercially available blocking solution (KPL Inc., Gaithersburg, Maryland). The finalised field IMS-LFD protocol was as illustrated in Fig. 1.

The $\mathrm{LOD}_{50 \%}$ of the field IMS-LFD was estimated to be $2.8 \times 10^{5} \mathrm{M}$. bovis cells $/ \mathrm{ml}$ faeces homogenate (1:10 dilution), so the detection sensitivity of the LFD was reduced when IMS preceded LFD detection, and faeces homogenates rather than PBS suspensions were being tested.

\section{Capability of the IMS-LFD test to detect $M$. bovis in badger faeces relative to the laboratory-based IMS-qPCR and IMS-culture tests}

A total of 441 badger faecal samples, collected from 110 main setts throughout Northern Ireland were tested. A mean of four faeces samples were collected from a single latrine at each main sett. Using the individual faecal sample as the unit of analysis, 78 faecal samples (18\%) tested positive with the field IMS-LFD test, 140 (32\%) tested positive by IMS-qPCR, and 64 (15\%) tested positive by IMS-culture, with only three faecal samples (0.7\%) testing positive by all three tests (Fig. 3a). Given the $\mathrm{LOD}_{50 \%}$ of the IMS-LFD assay applied to badger faeces indicated above, a positive result with this assay should indicate the presence of $\sim 10^{5} \mathrm{M}$. bovis cells $/ \mathrm{ml}$, which should be visible microscopically. Acid-fast bacteria attached to the magnetic beads were observed for several of the IMS-LFD 


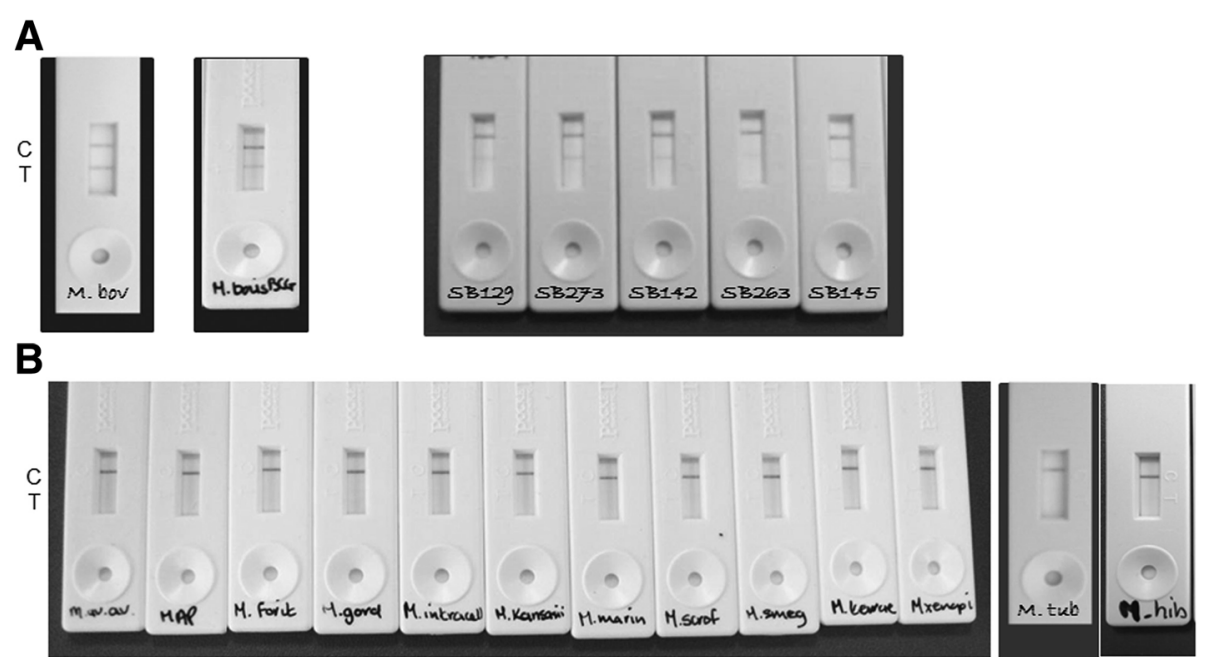

Fig. 2 Outcomes of specificity testing of the prototype lateral flow immunochromatographic device (LFD) demonstrating (a) the presence of a positive T-line with Mycobacterium bovis AF2122/97, M. bovis BCG NCTC 5692 and five other M. bovis spoligotypes and (b) only the presence of a C-line for all other Mycobacterium spp. tested

positive faecal samples, providing confirmation that high numbers of acid-fast bacteria (assumed to be $M$. bovis) were indeed present when an IMS-LFD positive result was obtained.

Field IMS-LFD results were compared with results of the two laboratory IMS-based methods; neither of which was being considered as the gold standard method for detecting $M$. bovis in faeces. There was no significant association between the results of field IMS-LFD, IMS-qPCR or IMS-culture $(P>0.05)$. Kappa statistics indicated 'poor' agreement between IMS-LFD and IMS-qPCR results (Kappa $=-0.009,95 \% \mathrm{CI}:-0.095$ to $0.077, p=0.838)$ and 'slight' agreement (Kappa $=0.045$,
95\% CI: -0.054 to $0.144, p=0.342)$ between IMS-LFD and IMS-culture results (Table 1A).

Estimation of the relative diagnostic specificity and sensitivity of the IMS-LFD assay by testing faeces from badgers of known infection status

The TB infection status of the 100 badgers from Woodchester Park that had contributed the faecal samples tested was only revealed to laboratory staff once results became available for the IMS-based tests. The badgers were categorised as putative 'TB infected' if a positive result had been obtained for Stat-Pak ${ }^{\bullet}$, culture or IFN- $\gamma$ EIA tests on any test occasion previously, and as putative 'non-

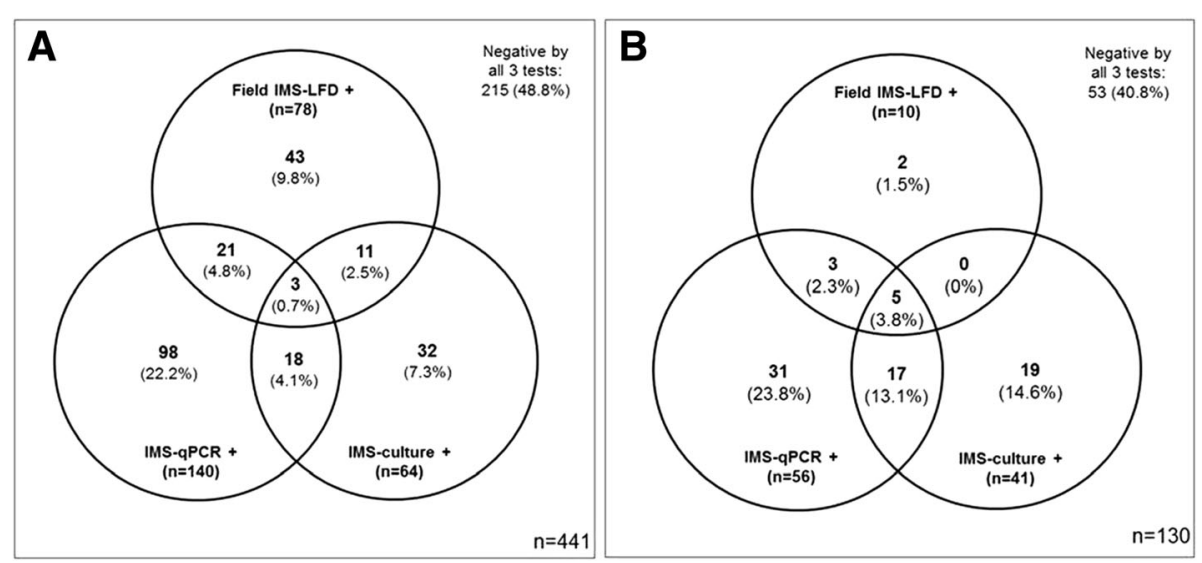

Fig. 3 Venn diagrams showing the numbers (percentage) of faeces samples that tested positive by field IMS-LFD, IMS-qPCR and IMS-culture from 441 badgers of unknown infection status collected from latrines at main setts in Northern Ireland (a) and from 100 live captured badgers and 30 captive putative TB negative badgers for which independent live animal diagnostic test data were available (b). Areas of overlap indicate positive test results in common 
Table 1 Cross-tabulation of field IMS-LFD, IMS-qPCR and IMS-culture results for 441 badger faecal samples freshly collected from latrines at main setts in Northern Ireland (A), and 130 faecal samples obtained from live captured or captive badgers of putative known TB infection status (B)

\begin{tabular}{|c|c|c|c|c|c|c|}
\hline \multicolumn{7}{|l|}{ (A) } \\
\hline \multirow[t]{2}{*}{ Field test result } & \multicolumn{6}{|c|}{ Laboratory-based test results: } \\
\hline & IMS-qPCR + & IMS-qPCR - & Kappa \pm SE (significance) & IMS- culture + & IMS- culture - & Kappa \pm SE (significance) \\
\hline IMS-LFD + & 24 & 54 & $-0.009 \pm 0.044$ & 14 & 64 & $0.045 \pm 0.051$ \\
\hline IMS-LFD - & 116 & 247 & $\left(p=0.838^{N S}\right)$ & 50 & 313 & $\left(p=0.342^{\mathrm{NS}}\right)$ \\
\hline Total & 140 & 301 & & 64 & 377 & \\
\hline \multicolumn{7}{|l|}{ (B) } \\
\hline \multirow[t]{2}{*}{ Field test result } & \multicolumn{6}{|c|}{ Laboratory-based test results: } \\
\hline & IMS-qPCR + & IMS-qPCR - & Kappa \pm SE (significance) & IMS- culture + & IMS- culture - & Kappa \pm SE (significance) \\
\hline IMS-LFD + & 8 & 2 & $0.129 \pm 0.056$ & 5 & 5 & $0.083 \pm 0.063$ \\
\hline IMS-LFD - & 48 & 72 & $\left(p=0.014^{*}\right)$ & 36 & 84 & $\left(p=0.083^{\mathrm{NS}}\right)$ \\
\hline Total & 56 & 74 & & 41 & 89 & \\
\hline
\end{tabular}

NS no significant association, ${ }^{*} P<0.05$, significant association at the $95 \%$ level

infected' if a negative result had been obtained for all three live animal tests on each previous testing occasion. Overall, there were 62 faecal samples from putative 'TB infected' and 38 from putative 'non-infected' badgers in this cohort. The field IMS-LFD tested positive for $10 \%$ of faecal samples obtained by enema from these 100 live captured badgers, whilst IMS-qPCR and IMS-culture tested positive for $56 \%$ and $41 \%$ of samples, respectively. Five (3.8\%) samples tested positive by all three IMS based tests (Fig. 3b). Two samples tested positive by the IMSLFD test and not by either of the other two IMS-based tests. All of the 30 faeces from putative 'non-infected' captive badgers tested negative by field IMS-LFD, IMSqPCR and IMS-culture, so no false positive IMS-LFD results were obtained. Overall, Kappa statistics indicated 'poor' agreement between IMS-LFD and IMS-qPCR results (Kappa $=0.129,95 \%$ CI: 0.019 to $0.238, p=0.014$ ) and also between IMS-LFD and IMS-culture results $($ Kappa $=0.083,95 \%$ CI: -0.056 to $0.221, p=0.083)$ for the 130 faeces samples tested (Table 1B).

Cross-tabulation of badger infection status (on the basis of prior live animal diagnostic test results) with IMS-LFD, IMS-qPCR and IMS-culture results (Table 2) enabled estimates of relative diagnostic specificity (Sp) and sensitivity (Se) to be obtained using McNemar's test. IMS-qPCR and IMS-culture had much higher diagnostic Se (0.581 and 0.436, respectively) than the IMS-LFD test (0.081) (Table 2). However, the IMS-LFD test had greater diagnostic Sp (0.926) than either IMS-qPCR (0.706) or IMS-culture (0.794) (Table 2).

\section{Discussion}

The IMS-LFD test described here was developed with a view to it being a rapid and non-invasive test to detect the presence of whole $M$. bovis cells in badger faeces. To our knowledge, the prototype LFD developed is the first of its kind. Other $M$. bovis LFDs are available commercially, but these detect either serum antibodies to $M$. bovis (BrockTB Stat-Pak ${ }^{\circledR}$ assay or DPP ${ }^{\circledR}$ CervidTB assay, both Chembio Diagnostic Systems, Inc., Medford, NY), or the MPT64 antigen secreted by members of the Mycobacterium tuberculosis complex, which includes $M$. bovis, in liquid culture (BD MGIT ${ }^{\mathrm{Tm}} \mathrm{TBc}$ Identification Test, Becton, Dickinson and Company, NJ; SD Bioline TB Ag MPT 64, Standard Diagnostics, Inc., Gyeonggi-do, Republic of Korea; Capilia TB-Neo kit, TAUNS Laboratories, Inc., Shizuoka, Japan). The unique specificity of the LFD developed during this study is due to the particular monoclonal antibody conjugated to gold nanoparticles and used as the detector reagent in the novel LFD; for commercial reasons, no further details of this antibody can be provided. The novel $M$. bovis-specific LFD was used in combination with a modified IMS technique (involving smaller magnetic beads) in order to capture and concentrate $M$. bovis cells from the faeces matrix and remove gross faecal components that could potentially block movement of the beads along the LFD.

When 441 badger faeces samples collected from latrines at main setts throughout Northern Ireland were tested by the IMS-LFD assay, IMS-qPCR and IMS-culture, $M$. bovis was detected in $18 \%, 32 \%$ and $15 \%$ of faecal samples respectively (Fig. 3a). There was little agreement between the results of the three IMS-based tests. Whilst the same faecal homogenate was tested by all three methods, different volumes of material were tested in the field (by IMS-LFD) and in the laboratory (by IMS-qPCR and IMS-culture). Automated IMS in the laboratory was restricted to analysing $1 \mathrm{ml}$ of sample whereas the field IMS was carried out on a 6-8 $\mathrm{ml}$ volume of filtered faecal homogenate. We estimate that there was the equivalent 
Table 2 Estimates of the diagnostic specificity (Sp) and sensitivity (Se) of the field IMS-LFD and laboratory-based IMS-qPCR and IMS-culture tests for M. bovis in faeces from 100 live captured badgers and 30 live captive badgers relative to live diagnostic test results, obtained using McNemar's test (95\% confidence intervals are indicated in parentheses)

\begin{tabular}{lccccc}
\hline Test result & Putative TB infected & Putative Non-infected & Total & Diagnostic sensitivity, Se & Diagnostic specificity, Sp \\
\hline IMS-LFD + & 5 & 5 & 10 & 0.081 & 0.926 \\
IMS-LFD - & 57 & 63 & 120 & $(0.027-0.178)$ & $(0.837-0.976)$ \\
IMS-qPCR + & 62 & 68 & 130 & & 0.706 \\
IMS-qPCR - & 36 & 20 & 56 & 0.581 & $(0.583-0.811)$ \\
IMS-culture + & 26 & 48 & 74 & $(0.449-0.705)$ & 0.794 \\
IMS-culture - & 62 & 68 & 130 & & $(0.678-0.882)$ \\
& 35 & 14 & 41 & $(0.310-0.567)$ & \\
\hline
\end{tabular}

of $\sim 250 \mathrm{mg}$ faeces per $80 \mu \mathrm{l}$ bead sample applied to the LFD after IMS in the field, $\sim 5 \mathrm{mg}$ faeces per IMSqPCR reaction, and $\sim 80 \mathrm{mg}$ faeces per IMS-culture (assuming no losses during sample processing), so the higher volume of sample used for the IMS-LFD assay may explain the few extra samples which tested positive by the field IMS-LFD test (Fig. 3a). That said, we estimated in preliminary studies [25] the $\mathrm{LOD}_{50 \%}$ of IMS-qPCR to be $1.7 \times 10^{4} \mathrm{M}$. bovis cells $/ \mathrm{ml}$ of faeces homogenate (or $1.7 \times 10^{5} \mathrm{M}$. bovis cells/g faeces), indicating that the IMS-qPCR method should theoretically have greater detection sensitivity than the field IMSLFD assay (which was found to have an $\mathrm{LOD}_{50 \%}$ of $2.8 \times 10^{5} \mathrm{M}$. bovis cells $/ \mathrm{ml}$ of faecal homogenate or $2.8 \times 10^{6} \mathrm{M}$. bovis cells/g faeces). This being the case, more $M$. bovis positive faecal samples should have been detected by IMS-qPCR than by IMS-LFD, which was the situation during this study. Furthermore, differently sized magnetic beads were used in the field and laboratory tests, because only the smaller $(300 \mathrm{~nm})$ coated beads would pass along the LFD. Despite being coated with the same $M$. bovis-specific binders, we know that the $300 \mathrm{~nm}$ Ademtech beads have slightly less capture capability than the $1 \mu \mathrm{m}$ MyOne Tosylactivated Dynabeads; this may also have influenced results obtained for the field IMS-LFD test compared to the laboratory IMSbased tests. We also suspect, but cannot demonstrate, that various environmental, logistical and practical considerations may have influenced the faecal sample preparation and IMS capture steps in the field, potentially leading to non-optimal performance of the IMS-LFD test. Some or all of these factors may have contributed to, and hence explain, the lack of agreement between field IMS-LFD and laboratory IMS-based test results. Test results for badger faeces collected from latrines at setts have demonstrated for the first time that the novel LFD is capable of detecting $M$. bovis cells in naturally contaminated badger faeces. However, due to the lack of agreement between results of the different IMS-based tests (Table 1A), with the lowest number of $M$. bovis positive samples being detected by IMS-LFD, results suggest that the novel IMS-LFD assay would be of limited use for badger TB surveillance purposes. Although the LFD was shown to have unique specificity for $M$. bovis (Fig. 2), the analytical sensitivity indicated by the $\mathrm{LOD}_{50 \%}$ of the combined IMS-LFD assay $\left(2.8 \times 10^{5} \mathrm{M}\right.$. bovis cells $/ \mathrm{ml}$ faecal homogenate $)$ means that only badgers shedding high numbers of $M$. bovis in their faeces would be detected, and infected badgers shedding lower numbers of $M$. bovis would test negative by the IMS-LFD assay. Given its reasonably high $\mathrm{LOD}_{50 \%}$, the novel IMS-LFD test may be more suited for applications where high numbers of $M$. bovis would be encountered, such as confirming the isolation of $M$. bovis from animal tissues in liquid cultures in the veterinary diagnostic laboratory context. This possibility is currently being investigated.

The ability of a novel diagnostic test to correctly identify infected and non-infected animals is an important consideration, so in order to determine the relative diagnostic sensitivity and specificity of the novel IMS-LFD assay, we tested 130 faeces samples from badgers for which independent diagnostic test results were available. Of these, the IMS-LFD tested positive in 10 (7.7\%) cases, whilst $56(43.0 \%)$ and $41(31.5 \%)$ samples tested positive by IMS-qPCR and IMS-culture, respectively (Fig. 3a). Test results for the subset of 30 faeces samples from putative TB negative captive badgers indicated that all samples were IMS-LFD, IMS-qPCR and IMS-culture negative; thus providing evidence of the high relative specificity of the novel IMS-LFD test, and indeed the other two IMS-based tests. The IMS-LFD test was found to have high diagnostic Sp (0.926) but low diagnostic Se (0.018), relative to the comparator live animal diagnostic tests, indicating an inability to detect low numbers of $M$. bovis cells in faeces, whereas IMS-qPCR and IMS-culture had lower diagnostic Sp (0.706 and 0.794, respectively) 
but higher diagnostic Se (0.581 and 0.436, respectively). It is acknowledged that potential mis-classification of the TB infection status of the badgers in our study due to false negative live animal test results may have impacted our estimates of the sensitivity and specificity of IMS-based tests (Table 2), so the latter should be treated with caution and only as relative measures of test performance.

The percentages of badger faeces samples from Woodchester Park testing M. bovis positive by IMS-qPCR and IMS-culture reported here appear relatively high. However 62 of the 100 faeces samples we tested were from putative 'TB infected' badgers with at least one previous positive live animal test result. Hence the $8.1 \%, 58 \%$ and $43.5 \%$ which tested IMS-LFD, IMS-qPCR and IMSculture positive, respectively, could be considered as the percentage of $M$. bovis faecal positives amongst putative 'TB infected' badgers only. However, it is notable that the presence of $M$. bovis was also indicated by one or more of the IMS-based tests in 25 (65.8\%) of 38 faeces samples from putative 'non-infected' badgers (Fig. 4). This was unexpected since faecal shedding of $M$. bovis by badgers has been generally associated with advanced disease [9], and may be intermittent and heterogeneous [12]. It is possible that some of these putative 'non-infected' badgers were misclassified as such owing to limitations in live test performance [31]. Also, in the cases of the IMS-LFD and

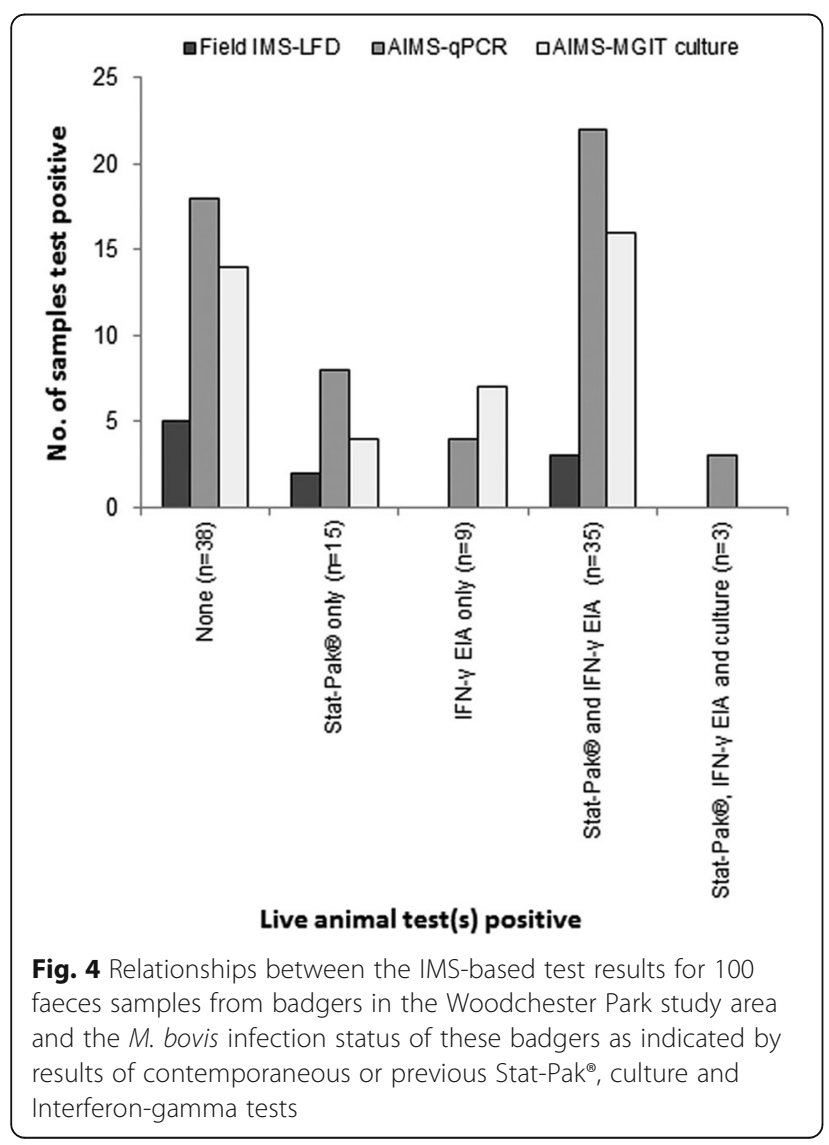

IMS-qPCR tests, positive results could have arisen from the detection of dead $M$. bovis cells originating from the environment and not indicative of infection, although an IMS-culture test positive result requires mycobacterial growth and so indicates the presence of live viable bacteria. In conclusion, the present study was not designed to provide estimates of the proportion of badgers shedding viable $M$. bovis in their faeces, rather to test the performance of novel non-invasive tests, nevertheless our results suggest that further investigation of the extent of faecal shedding in badgers is warranted.

In this study, $M$. bovis cells were concentrated and selectively captured by IMS from several millilitres of an homogenate of approximately $1 \mathrm{~g}$ faeces in PBS in advance of detection by LFD, qPCR and culture during this study; a testing approach that has never been used before on badger faeces. The traditional badger faeces testing approach would be to decontaminate faecal suspensions in PBS with $0.75 \%$ cetylpyridinium chloride overnight prior to culture in liquid and/or solid media [10] or to extract total community DNA from approximately $0.1 \mathrm{~g}$ faeces prior to M. bovis-specific qPCR [12]. By testing a larger quantity of faeces, selectively isolating M. bovis cells prior to DNA extraction as well as separating them from PCR inhibitors by IMS, and not employing chemical decontamination which is known to have an adverse impact on the viability of $M$. bovis cells [32, 33], the chances of detection/isolation of $M$. bovis from faeces were likely to have been improved. The three IMS-based methods do not have equivalent detection sensitivity, so it is not surprising that the least sensitive test (IMS-LFD assay) detected lower numbers of $M$. bovis positive faecal samples than both IMS-qPCR and IMS-culture (Fig. 3a and b). Stewart et al. [22] previously reported that IMS-qPCR and IMS-culture improved detection rates of $M$. bovis in cattle lymph nodes compared to direct qPCR and chemical decontamination and culture. Results of the present study suggest that a similar boost in $M$. bovis detection rates has generally been observed by applying these IMS-based methods to test badger faeces. IMS applied to faeces samples in advance of application to the LFD should in theory have improved the detection sensitivity of the LFD, since $M$. bovis cells were concentrated from $6 \mathrm{ml}$ faecal homogenate into 200 ul before testing. In reality, despite the fact that smaller $(300 \mathrm{~nm})$ paramagnetic beads were employed, samples after field IMS on faeces samples did not always run easily along the LFD due to bead aggregation. Consequently, detection sensitivity actually decreased and this was not simply due to a sample matrix effect. Results of a subsequent ring trial study to compare IMS-LFD and direct LFD with other methods for detecting $M$. bovis in badger faeces showed that fewer positive results were obtained after IMS than by direct LFD testing [34]. 


\section{Conclusions}

A novel prototype $M$. bovis-specific LFD was successfully developed which, in combination with IMS, was shown to be capable of detecting $M$. bovis cells in some badger faeces samples tested during this study. However, there was generally little agreement between results of field IMS-LFD and laboratory-based IMS-qPCR and IMS-culture tests; the IMS-LFD assay detected the lowest percentage of $M$. bovis-infected badger faecal samples, reflective of its higher $\mathrm{LOD}_{50 \%}$. The novel IMS-LFD test was shown to have relatively high diagnostic $\mathrm{Sp}(0.926,95 \% \mathrm{CI}$ : $0.837-0.976)$ but low diagnostic sensitivity (0.081, 95\% CI: $0.027-0.178)$, relative to a suite of other live animal diagnostic tests. In light of these findings, the potential of the novel IMS-LFD test for M. bovis in badger faeces as a non-invasive field test for badger surveillance purposes would appear to be potentially limited to detecting badgers shedding high numbers of $M$. bovis $\left(\geq 10^{5} \mathrm{CFU} / \mathrm{g}\right)$ in their faeces. Alternative applications for the novel $M$. bovis-specific LFD are currently being explored.

\begin{abstract}
Abbreviations
95\% Cl: 95\% Confidence Interval; CFU: Colony-forming units, expressed per $\mathrm{ml}$ or g; C-line: Control line on LFD; DNA: Deoxyribonucleic acid; IFN- $\gamma$ EIA: Interferon Gamma Enzyme Immuno Assay; IgG: Immunoglobulin G; IMS: Immunomagnetic Separation; IMS-LFD: Immunomagnetic separation followed by lateral flow device test; IMS-culture: Immunomagnetic separation followed by culture in BD MGIT culture medium; IMS-qPCR: Immunomagnetic separation followed by quantitative PCR; LFD: Lateral flow device; LOD $50 \%$ : 50\% limit of detection; Mab: Monoclonal antibody; Pab: Polyclonal antibody; PBS: Phosphate buffered saline, pH 7.2; PBS-T: PBS containing 0.05\% Tween 20; PCR: Polymerase chain reaction; qPCR: Quantitative PCR; RTA: Road traffic accidents; Se: Diagnostic sensitivity; Sp: Diagnostic specificity; TB: Tuberculosis, in this case bovine tuberculosis; T-line: Test line on LFD
\end{abstract}

\section{Acknowledgements}

We would wish to thank Dr. Jim McNair, Veterinary Sciences Division, Agri-Food and Biosciences Institute for Northern Ireland, for providing access to one of the antibodies evaluated as part of this study, all the Northern Ireland farmers/ landowners who permitted access to their land for collection of badger faeces, and the field team of the National Wildlife Management Centre at Woodchester Park for the live capture and sampling of badgers in their study area.

\section{Availability of data and materials}

All data generated or analysed during this study are included in this published article. For Intellectual Property reasons, details of materials and reagents involved in production of the novel $M$. bovis-specific lateral flow device cannot be shared.

\section{Funding}

This research study was funded by the Department of Environment, Food and Rural Affairs (Defra), United Kingdom (Project SE3271). Neil Reid was supported by the Natural Heritage Research Partnership between the Northern Ireland Environment Agency and Quercus, Queen's University Belfast. We also thank Defra for funding the Woodchester Park study.

\section{Authors' contributions}

LDS processed faecal samples for IMS-qPCR and IMS-culture. NT and PM developed the prototype LFD using reagents supplied by LDS and IRG. JMA collected badger faeces samples from NI setts and performed field IMS-LFD. NR and WIM advised JMA on locations of badger setts throughout NI and sample collection. RN assisted LDS to perform qPCR on all samples. RJD and RA contributed faecal samples from badgers and associated live animal test results. NR and IRG performed statistical analysis of results. LDS and IRG conceived and designed the study, prepared tables and figures, and wrote the manuscript All authors contributed to the final version of the manuscript.

\section{Competing interests}

We declare no competing interests, financial or otherwise.

\section{Consent for publication}

Not applicable.

\section{Ethics approval}

Ethics approval was not required for Northern Ireland part of the study, as only badger faeces not live badgers were involved. The capture of wild badgers at Woodchester Park is undertaken under licence from Natural England, and sampling under anaesthesia is conducted under a UK Home Office Project Licence. All live animal work was approved by the Animal and Plant Health Agency (APHA) Animal Welfare Ethical Review Process. All captive animal procedures were covered by licenses issued by the UK Home Office under the Animals (Scientific Procedures) Act 1986, and also approved by the Animal Welfare and Ethical Review Board at APHA.

\section{Publisher's Note}

Springer Nature remains neutral with regard to jurisdictional claims in published maps and institutional affiliations.

\section{Author details}

${ }^{1}$ Institute for Global Food Security, School of Biological Sciences, Queen's University Belfast, Belfast, Northern Ireland, UK. Forsite Diagnostics Limited (now Abingdon Health), National Innovation Campus, Sand Hutton, York, UK. ${ }^{3}$ Quercus, School of Biological Sciences, Belfast, Northern Ireland, UK.

${ }^{4}$ National Wildlife Management Centre, Animal and Plant Health Agency, Woodchester Park, Nympsfield, Gloucestershire, UK. ${ }^{5}$ Animal and Plant Health Agency, Weybridge, New Haw, Addlestone, Surrey, UK.

Received: 28 February 2017 Accepted: 4 May 2017

Published online: 12 May 2017

\section{References}

1. Gallagher J, Clifton-Hadley RS. Tuberculosis in badgers: a review of the disease and its significance for other animals. Res Vet Sci. 2000:69:203-17.

2. Wilson GJ, Carter SP, Delahay RJ. Advances and prospects for management of TB transmission between badgers and cattle. Vet Microbiol. 2011;151:43-50.

3. Independent Study Group on Cattle TB. Bovine TB: The Scientific Evidence. 2007. [ http://www.bovinetb.info/docs/final_report.pdf]. Accessed 8 May 2017.

4. Wright DM, Reid N, Montgomery WI, Allen AR, Skuce RA, Kao RR. Herd-level bovine tuberculosis risk factors: assessing the role of low-level badger population disturbance. Sci Rep. 2015;5:13062.

5. Skuce RA, Neill SD. Molecular epidemiology of Mycobacterium bovis: exploiting molecular data. Tuberculosis. 2001:81:169-75.

6. Haddad N, Masselot M, Durand B. Molecular differentiation of Mycobacterium bovis isolates: review of main techniques and applications. Res Vet Sci. 2004;76:1-18.

7. Olea-Popelka FJ, Flynn O, Costello E, McGrath G, Collins JD, O'Keeffe J, Kelton DF, Berke O, Martin SW. Spatial relationship between Mycobacterium bovis strains in cattle and badgers in four areas in Ireland. Prev Vet Med. 2005:71:57-70.

8. Little TWA, Naylor PF, Wilesmith JW. Laboratory study of Mycobacterium bovis infection in badgers and calves. Vet Rec. 1982;111:550-7.

9. Clifton-Hadley RS, Wilesmith JW, Stuart FA. Mycobacterium bovis in the European badger (Meles meles): epidemiologic findings in tuberculous badgers from a naturally infected-population. Epidemiol Infect. 1993;111:9-19.

10. Corner LAL, O'Meara D, Costello E, Lesellier S, Gormley E. The distribution of Mycobacterium bovis infection in naturally infected badgers. Vet J. 2012;194:166-72.

11. Hutchings MR, Service KM, Harris S. Defecation and urination patterns of badgers Meles meles at low density in south west England. Acta Theriol. 2001;46:87-96.

12. King HC, Murphy A, James P, Travis E, Porter D, Hung Y-J, Sawyer J, Cork J, Delahay RJ, Gaze W, Cortenay O, Wellington EM. The variability and seasonality of the environmental reservoir of Mycobacterium bovis shed by wild European badgers. Sci Rep. 2015;5:12318.

13. Wellington EM, Courtenay O. Badgers and bovine TB: how can environmental microbiology help? Microbiol Today. 2014;41:143-4. 
14. Young JS, Gormley E, Wellington EMH. Molecular detection of Mycobacterium bovis and Mycobacterium bovis BCG (Pasteur) in soil. Appl Environ Microbiol. 2005;71:1946-52

15. Courtenay O, Reilly LA, Sweeney FP, Hibberd V, Bryan S, Ul-Hassan A, Newman C, Macdonald DW, Delahay RJ, Wilson GJ, Wellington EMH. Is Mycobacterium bovis in the environment important for the persistence of bovine tuberculosis? Biol Lett. 2006;2:460-2.

16. Cheeseman CL, Wilesmith JW, Stuart FA. Tuberculosis: the disease and its epidemiology in the badger: a review. Epidemiol Infect. 1989;103:113-25.

17. Abernethy DA, Denny GO, Menzies FD, McGuckian P, Honhold N, Roberts AR. The Northern Ireland programme for the control and eradication of Mycobacterium bovis. Vet Microbiol. 2006;112:231-7.

18. Abernethy DA, Walton E, Menzies F, Courcier E, Robinson P. Mycobacterium bovis surveillance in European badgers (Meles meles) killed by vehicles in Northern Ireland: an epidemiological evaluation. 2011. [https://www.daerani.gov.uk/sites/default/files/publications/dard/m-bovis-surveillanceeuropean-badgers-rta-epi-eval.pdf]. Accessed 8 May 2017.

19. Krebs J, Anderson R, Clutton-Brock T, Morrison I, Young D, Donnelly C. Bovine Tuberculosis in cattle and badgers.Report by the Independent Scientific Review Group. Ministry of Agriculture, Fisheries and Food, London. 1997. [http://www.bovinetb.info/docs/krebs.pdf]. Accessed 8 May 2017.

20. Delahay RJ, Walker N, Smith GS, Wilkinson D, Clifton-Hadley RS, Cheeseman CL, Tomlinson AJ, Chambers MA. Long-term temporal trends and estimated transmission rates for Mycobacterium bovis infection in an undisturbed high density badger population. Epidemiol Infect. 2013;141:1445-56.

21. Stewart LD, McNair J, McCallan L, Thompson S, Kulakov L, Grant IR. Production and evaluation of antibodies and phage display-derived peptide ligands for immunomagnetic separation of Mycobacterium bovis. J Clin Microbiol. 2012;50:1598-605.

22. Stewart LD, McNair J, McCallan L, Grant IR. Improved detection rates of Mycobacterium bovis infection in bovine lymph node tissue using immunomagnetic separation (IMS)-based methods. PLoS One. 2013;8:e58374.

23. $A O A C$ International. Final report and executive summaries from the $A O A C$ International. Presidential Task Force on Best Practices in Microbiological Methodology 2006. [http://www.fda.gov/downloads/Food/FoodScienceResearch/ UCM088702.pdf]. Accessed 8 May 2017.

24. Department of Environment, Food and Rural Affairs.EVID4 Final Report on Defra Project SE3271: Development and field validation of a rapid immunomagnetic separation-lateral flow (IMS-LF) test for detecting Mycobacterium bovis infection in badgers and/or badger setts. 2014. [http:// randd.defra.gov.uk/Document.aspx?Document=13141_SE3271.pdf]. Accessed 8 May 2017.

25. Reid N, Etherington TR, Wilson G, Montgomery WI, McDonald RA. Monitoring and population estimation of the European badger Meles meles in Northern Ireland. Wildlife Biol. 2011;18:46-57.

26. Sweeney FP, Courtenay O, Hibberd V, Hewinson RG, Reilly LA, Gaze WH, Wellington EMH. Environmental monitoring of Mycobacterium bovis in badger feces and badger sett soil by real-time PCR, as confirmed by immunofluorescence, immunocapture, and cultivation. Appl Environ Microbiol. 2007;73:7471-3.

27. Travis ER, Gaze WH, Pontiroli A, Sweeney FP, Porter D, Mason S, Keeling MJC, Jones RM, Sawyer J, Aranaz A, Castellanos Rizaldos E, Cork J, Delahay RJ, Wilson GJ, Hewinson RG, Courtenay O, Wellington EMH. An interlaboratory validation of a real time PCR assay to measure host excretion of bacterial pathogens, particularly of Mycobacterium bovis. PLoS One. 2011;6:e27369.

28. Zumárraga MJ, Meikle V, Bernardelli A, Abdala A, Tarabla H, Romano MI, Cataldi A. Use of touch-down polymerase chain reaction to enhance the sensitivity of Mycobacterium bovis detection. J Vet Diag Invest. 2005;17:232-8.

29. Dalley D, Dave D, Lesellier S, Palmer S, Crawshaw T, Hewinson RG, Chambers M. Development and evaluation of a gamma-interferon assay for tuberculosis in badgers (Meles meles). Tuberculosis. 2008:88:235-43.

30. Landis JR, Koch GG. The measurement of observer agreement for categorical data. Biometrics. 1977;33:159-74.

31. Drewe JA, Tomlinson A, Walker NJ, Delahay RJ. Diagnostic accuracy and optimal use of three tests for tuberculosis in live badgers. PLoS One. 2010; 5(6):e11196.

32. Corner LAL, Trajstmann AC. An evaluation of 1-hexadecylpyridinium chloride as a decontaminant in the primary isolation of Mycobacterium bovis from bovine lesions. Vet Microbiol. 1988;18:127-34.
33. Ambrosio SR, de Deus Oliveira EM, Rosales Rodriguez CA, Ferreira Neto JS, Amaku M. Comparison of three decontamination methods for Mycobacterium bovis isolation. Braz J Microbiol. 2008;39:241-4.

34. Department of Environment, Food and Rural Affairs. Final Project Report SE3289: A study to comparatively assess diagnostic methods for detection of M. bovis in badger faeces. London: Department for Environment, Food and Rural Affairs; 2015. [http://randd.defra.gov.uk/Document.aspx?Document= 13243_SE3289.pdf]. Accessed 8 May 2017.

\section{Submit your next manuscript to BioMed Central and we will help you at every step:}

- We accept pre-submission inquiries

- Our selector tool helps you to find the most relevant journal

- We provide round the clock customer support

- Convenient online submission

- Thorough peer review

- Inclusion in PubMed and all major indexing services

- Maximum visibility for your research

Submit your manuscript at www.biomedcentral.com/submit
C Biomed Central 